\title{
STABILIZING TENSOR PRODUCTS
}

\author{
HAROLD M. HASTINGS 1
}

\begin{abstract}
Let $C$ be a symmetric monoidal category with a suspension, and let $S C$ be the resulting stable category. We shall give necessary and sufficient conditions for extending the symmetric monoidal structure to a monoidal structure on $S C$. These imply that the usual smash product on finite pointed CW complexes cannot be extended to a smash product (with $S^{0}$ as unit) on finite spectra, hence not on Boardman spectra. This confirms a conjecture of Alex Heller.
\end{abstract}

1. Introduction. Let $(C, \otimes, U)$ be a symmetric monoidal category (Eilenberg and Kelly [4, pp. 472, 512]). Here $\otimes$ is the tensor product, and $U$ the unit; $\otimes$ is an associative, commutative and "unitary" functor $C \times C \rightarrow C$. The standard example has $U$ a commutative ring, with identity, $C$ the category of $U$-modules, and $\otimes$ the usual tensor product.

If $C$ also admits a suspension, we may form the corresponding SpanierWhitehead [9] category SWC and universal stable category SC (Heller [6]). There are functors $C \rightarrow S W C \rightarrow S C$, the latter a full inclusion. See $\$ 2$.

In $\$ 3$ we shall give a simple necessary condition to extend the symmetric monoidal structure to SWC. This condition is motivated by the wellknown fact: a category of modules over a ring with identity $R$ admits a tensor product if and only if $R$ is commutative. As a consequence, the usual symmetric monoidal structure on the category $F$ of finite pointed $C W$ complexes and pointed continuous maps (here $X \otimes Y=X \wedge Y=X \times Y / X \vee Y, U=S^{0}$ ) cannot be extended to $S W F$; hence not to $S F$. This confirms a conjecture of Alex Heller.

In $\S 4$ we shall show that if $\Sigma=? \otimes S^{1}$ for some $S^{1}$ in $C$, then our necessary condition is also sufficient to extend the symmetric monoidal structure to SWC. Extension to $S C$ will then be automatic.

Presented to the Society, October 27, 1973; received by the editors January 28,1974 .

AMS (MOS) subject classifications (1970). Primary 18D10, 55B20; Secondary 55D99, 55E10, 55E 15, 55J99.

Key words and phrases. Smash product, stable smash product, SpanierWhitehead category, stable category, Boardman spectra, monoidal category, symmetric monoidal category, category with suspension.

1 Partially supported by a NSF Institutional Grant. 
Two applications, the second well known, will be given. In $\$ 5$ we shall extend the usual symmetric monoidal structure on $H F$ ( $H$ denotes homotopy category) to $S H F$ ( $\cong H S F$ ). Boardman [2], [10], Adams [1], Puppe [8], May [7], and we [5] have given ad hoc constructions of a symmetric monoidal . structure on Boardman's stable homotopy category $H B . B$ is the c-completion [6] of $S F$. We shall briefly compare these constructions.

In $\$ 6$, we shall show that the usual symmetric monoidal structure on the category of chain complexes with translation suspension can be extended to the corresponding stable category (\$6).

Acknowledgement. We wish to thank Alex Heller for helpful discussions during the preparation of this work.

2. Review of stable categories. We shall need the following definitions, due to Heller [6], except as noted.

Call a category $C$ with endofunctor $\Sigma$ a category with suspension. We shall use $\Sigma$ generically to denote suspensions. Such a category is called stable if $\Sigma$ is an automorphism. A functor $T$ between categories with suspension is called stable if $T \Sigma=\Sigma T$.

To each category $C$ with suspension, Heller associates a universal stable category [6, Proposition 1.1] SC. Objects of $S C$ are pairs $(X, m)$, where $X$ is an object of $C$, and $m$ is an integer. Morphisms are given by

$$
S C((X, m),(Y, n))=\operatorname{colim} C\left(\Sigma^{m+k} X, \Sigma^{n+k} Y\right)
$$

where $k$ ranges over any unbounded monotone sequence of integers. See, e.g., the proof of Theorem 4.

$S C$ admits a suspension given by $\Sigma(X, m)=(X, m+1)$. There is a functor $S: C \rightarrow S C$; on objects $S X=(X, 0)$. We shall sometimes identify $X$ with $(X, 0)$. Note that $S \Sigma \cong \Sigma S$.

Finally, the Spanier-Whitehead category [9] SWC is the full subcategory of $S C$ whose objects are in $C$ (precisely, in the image of $S$ ). $S$ factors through $S W C$.

3. The necessary condition and some consequences. Let $(D, \otimes, U)$ be a (not necessarily symmetric) monoidal category. That is, $\otimes$ need not be commutative.

Theorem 1. $D(U, U)$ is a commutative monoid.

Proof. We shall use the following part of the monoidal structure [4, p. 472], to show that any two maps in $D(U, U)$ commute. For any $X$ in $D$, 
there are natural right and left unit isomorphisms

$$
r_{X}: X \otimes U \rightarrow X, \quad l_{X}: U \otimes X \rightarrow X
$$

If $X=U, r_{U}=l_{U}$; call this map $u$. Hence any map $f$ in $D(U, U)$ factors as

$$
f=u(f \otimes U) u^{-1}=u(U \otimes f) u^{-1}
$$

Let $f, g \in D(U, U)$. Then

$$
\begin{aligned}
f g & =u(f \otimes U) u^{-1} u(U \otimes g) u^{-1}=u(f \otimes U)(U \otimes g) u^{-1} \\
& =u(f \otimes g) u^{-1}=u(U \otimes g)(f \otimes U) u^{-1} \\
& =u(U \otimes g) u^{-1} u(f \otimes U) u^{-1}=g f,
\end{aligned}
$$

as required.

Corollary 2. The following (symmetric) monoidal structures cannot be extended from the indicated categories to their Spanier-Whitehead categories, or universal stable categories.

(a) $C=F$, the category of finite pointed $C W$ complexes are continuous pointed maps, $\otimes=\wedge, U=S^{0}, \Sigma X=X \wedge S^{1}$.

(b) $C$ is the category of finite dimensional vector spaces and isomorphisms (or any larger category of vector spaces) over a field $F, \otimes$ is the usual direct sum $\oplus, U=0, \Sigma X=X \oplus F$.

(c) $C$ as in (b), $\otimes$ is the usual tensor product, $U=F, \quad \Sigma X=X \oplus F$.

For (a), let the symmetric group $\mathcal{S}_{n}$ act on $S^{2 n}$ by permuting factors of $S^{2} \wedge \ldots \wedge S^{2}$. Define inclusions $\delta_{n} \subset \mathcal{S}_{n+1}$ by "leaving the last letter fixed." Let $\mathcal{S}_{\infty}=\operatorname{colim} \mathcal{S}_{n}$. There is an induced inclusion of monoids

$$
\S_{\infty} \subset S W F\left(S^{0}, S^{0}\right)=\operatorname{colim} F\left(S^{2 n}, S^{2 n}\right)
$$

hence $S W F\left(S^{0}, S^{0}\right)$ is not abelian. Note that "signs" are not involved.

Proofs of (b) and (c) are similar. These examples are motivated by topological reduced $K_{F}$ theory.

4. Sufficient conditions. Let $(C, \otimes, U)$ be a symmetric monoidal category. Fix some object $S^{1}$ in $C$, and define a suspension by $\Sigma X=X \otimes S^{1}$.

We can then give a rough converse to Theorem 1 on extending the symmetric monoidal structure to SWC and SC.

Let $S^{0}=U$. For $n \geq 1$, let $S^{n}=\Sigma S^{n-1}=S^{n-1} \otimes S^{1}$. 
Let $\mathbb{Q}_{n}$ denote the alternating group on $n$ letters, the commutator subgroup of $\mathfrak{S}_{n}$. See Corollary 2, verification of (a). Regard $\mathbb{A}_{n} \subset \mathbb{Q}_{n+1}$ by "leaving the last letter fixed." Let $\mathfrak{Q}_{\infty}=$ colim $\mathfrak{Q}_{n}$; then $\mathfrak{Q}_{\infty}$ is the commutator subgroup of $\mathcal{S}_{\infty}$.

Definition 3. The standard action of $\mathcal{S}_{\infty}\left(\right.$ and $\left.\mathbb{A}_{\infty}\right)$ on $S^{0}$, a monoid homomorphism $\mathbb{Q}_{\infty} \subset \mathcal{S}_{\infty} \rightarrow \operatorname{SWC}\left(S^{0}, S^{0}\right)$, is the colimit of the homomorphisms $\mathbb{Q}_{n} \subset \mathcal{S}_{n} \rightarrow C\left(S^{n}, S^{n}\right)$ induced by permuting factors of $S^{n}=S^{1} \otimes \cdots \otimes S^{1}$.

Theorem 4. The following conditions on a symmetric monoidal category $\left(C, \otimes, S^{0}\right)$ with suspension $\Sigma=? \otimes S^{1}$ are equivalent.

(a) $S W C\left(S^{0}, S^{0}\right)$ is a commutative monoid.

(b) $\mathbb{Q}_{\infty}$ acts trivially on $S^{0}$.

(c) The symmetric monoidal structure on $C$ can be extended to SC $(S: C \rightarrow S C$ is a symmetric monoidal functor [4, pp. 473, 512]).

(d) The symmetric monoidal structure can be extended to SWC.

Proof. (a) $\Rightarrow$ (b) since $\mathbb{Q}_{\infty}$ is the commutator subgroup of $\mathcal{S}_{\infty}$.

For $(\mathrm{b}) \Rightarrow(\mathrm{c})$, define $\otimes$ on objects by $(X, m) \otimes(Y, n)=(X \otimes Y, m+n)$.

We shall define $\otimes$ on maps "up to choice" and then show that the definition is independent of choice. Let $f:(X, m) \rightarrow\left(X^{\prime}, m^{\prime}\right)$ and $g:(Y, n) \rightarrow$ $\left(Y^{\prime}, n^{\prime}\right)$ be maps in SC. Choose representatives of the form

$$
f^{\prime}: X \otimes S^{m+2 k} \rightarrow X^{\prime} \otimes S^{m^{\prime}+2 k}, \quad g^{\prime}: Y \otimes S^{n+2 l} \rightarrow Y^{\prime} \otimes S^{n^{\prime}+2 l} .
$$

Let $\left(f^{\prime} \otimes g^{\prime}\right)_{*}$ be the composite

$$
\begin{aligned}
& X \otimes Y \otimes S^{m+n+2 k+2 l} \cong X \otimes Y \otimes S^{m} \otimes S^{n} \otimes S^{2 k} \otimes S^{2 l} \\
& \rightarrow X \otimes S^{m} \otimes S^{2 k} \otimes Y \otimes S^{n} \otimes S^{2 l} \\
& \stackrel{f^{\prime} \otimes g^{\prime}}{\longrightarrow} X^{\prime} \otimes S^{m^{\prime}} \otimes S^{2 k} \otimes Y^{\prime} \otimes S^{n^{\prime}} \otimes S^{2 l} \\
& \rightarrow X^{\prime} \otimes Y^{\prime} \otimes S^{m^{\prime}} \otimes S^{n^{\prime}} \otimes S^{2 k} \otimes S^{2 l} \\
& \cong X^{\prime} \otimes Y^{\prime} \otimes S^{m^{\prime}+n^{\prime}+2 k+2 l} \text {. }
\end{aligned}
$$

Finally, let $f \otimes g$ be the image of $\left(f^{\prime} \otimes g^{\prime}\right)_{*}$ in

$$
S C\left((X \otimes Y, m+n),\left(X^{\prime} \otimes Y^{\prime}, m^{\prime}+n^{\prime}\right)\right)
$$

We must now show that $f \otimes g$ is well defined. Let $f^{\prime \prime}, g^{\prime \prime}$ be other representatives for $f, g$, respectively. Then for sufficiently large $N$, suit- 
able $K$ and $L$, and a suitable even permutation $\pi$ of factors of $S^{m+n+N}=$ $S^{1} \otimes \cdots \otimes S^{1}$, the following diagram commutes.

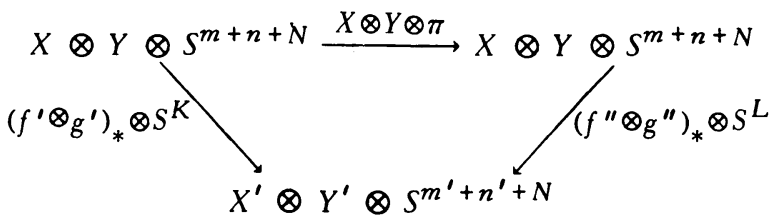

Since $\mathbb{A}_{\infty}$ acts trivially on $S^{0}$, then $\left(f^{\prime} \otimes g^{\prime}\right)_{*}$ and $\left(f^{\prime \prime} \otimes g^{\prime \prime}\right)_{*}$ have the same image in $S C\left((X \otimes Y, m+n),\left(X^{\prime} \otimes Y^{\prime}, m^{\prime}+n^{\prime}\right)\right)$, as required.

The axioms for $\left(S C, \otimes, S^{0}\right)$ to be a symmetric monoidal category and for $S$ to be a symmetric monoidal functor [4, pp. 472-473, 512] are easy to verify. For example, to prove coherent associativity of $\otimes$ on $S C$, let $a:(X \otimes Y) \otimes Z \rightarrow X \otimes(Y \otimes Z)$ be the associativity isomorphism on $C$. The associativity morphism on $S C$ is the composite

$$
\begin{aligned}
((X, m) \otimes(Y, n)) \otimes(Z, p) & =((X \otimes Y) \otimes Z, m+n+p) \\
& \stackrel{a_{*}}{\longrightarrow}(X \otimes(Y \otimes Z), m+n+p) \\
& =(X, m) \otimes((Y, n) \otimes(Z, p)) .
\end{aligned}
$$

It is easy to see that this is a coherent isomorphism. Remaining details are omitted.

(c) $\Rightarrow$ (d) since SWC is a full subcategory of SC which contains the unit and is closed under $\otimes$.

(d) $\Rightarrow$ (a) by Theorem 1 .

Remarks 5. The above tensor product on SC is separately stable (\$2) in each variable. There is at most one such stable extension.

The following simple condition on a symmetric monoidal category $\left(C, \otimes, S^{0}\right)$ with suspension ? $\otimes S^{1}$ will be shown to imply Theorem $4(\mathrm{~b})$. Let

$$
\tau: S^{3} \cong S^{1} \otimes S^{2} \rightarrow S^{2} \otimes S^{1} \cong S^{3}
$$

denote the transposition. If $\tau=S^{3}$, we shall say that $\tau$ acts trivially. Since the 3-cycles in $\mathbb{Q}_{\infty}$ generate $\mathbb{Q}_{\infty}$, we have

Proposition 6. If $\tau$ acts trivially, then $\mathbb{A}_{\infty}$ acts trivially on $S^{0}$; hence the symmetric monoidal structure can be extended.

Conversely, if $C, \Sigma$ satisfy the following weak Freudenthal condition, 
that $\Sigma: C\left(S^{n}, S^{n}\right) \rightarrow C\left(S^{n+1}, S^{n+1}\right)$ is a monomorphism for $n \geq 3$, and if $\mathbb{Q}_{\infty}$ acts trivially on $S^{0}$, then $\tau$ acts trivially.

5. The smash product for finite spectra. The homotopy category of finite pointed $\mathrm{CW}$ complexes, $H F$, inherits a symmetric monoidal structure and suspension from $F$ (see Corollary 2(a)).

Theorem 7. There are induced symmetric monoidal structures on SWHF and SHF.

Proof. Since the transposition $S^{1} \wedge S^{2} \rightarrow S^{2} \wedge S^{1}$ is homotopic to the identity on $S^{3}$; this is immediate from Proposition 6 .

This construction cannot be extended to Boardman's stable homotopy category $H B$, since $H B$ is the homotopy category of the c-completion [6] of $S F$ (the order of these constructions cannot be reversed), and our smash product is not even defined on $S F$.

To construct the smash product on $H B$ requires choosing nonassociative smash products on SF (the choices are similar to those of Theorem 4, "(b) $\Rightarrow(c)$ ") [1], [2], [5], [9], or a different construction of $B$ (and choices) [7], [8]. The former nonassociative smash products project to our smash product on HSF. The latter approach is similar to Boardman and Vogt's theory of infinite loop spaces [3], and induces the same smash product on HSF.

6. An example: chain complexes. The category $C$ of nonnegatively graded chain complexes over a commutative ring $R$ is a well-known example of a symmetric monoidal category, see e.g. [4, p. 558]. If $\left(X^{\prime}, d^{\prime}\right)$ and $\left(X^{\prime \prime}, d^{\prime \prime}\right)$ are chain complexes, define their tensor product $(X, d)$ by

$$
X_{n}=\bigoplus_{i+j=n} X_{i}^{\prime} \otimes X_{j}^{\prime \prime}, \quad d \mid X_{i}^{\prime} \otimes X_{j}^{\prime \prime}=d_{i}^{\prime} \otimes X_{j}^{\prime \prime}+(-1)^{i} X_{i}^{\prime} \otimes d_{j}^{\prime \prime} .
$$

The unit $S^{0}$ has $S_{0}^{0}=R, S_{i}^{0}=0$ otherwise, and 0 differential. It is clear that this yields a monoidal structure; the symmetry

$$
c:\left(X^{\prime}, d^{\prime}\right) \otimes\left(X^{\prime \prime}, d^{\prime \prime}\right) \rightarrow\left(X^{\prime \prime}, d^{\prime \prime}\right) \otimes\left(X^{\prime}, d^{\prime}\right)
$$

is given on $X_{i}^{\prime} \otimes X_{j}^{\prime \prime}$ by $c\left(x^{\prime} \otimes x^{\prime \prime}\right)=(-1)^{i j} x^{\prime \prime} \otimes x^{\prime}$.

The "translation" suspension $\Sigma$ on $C$ may also be given by letting $S^{1}$ be the chain complex which is $R$ in degree 1, 0 otherwise, and has 0 differential. Then $\Sigma X=X \otimes S^{1}$. 
Since $S W C \cong C, S W C$, and, hence, $S C$ (by Theorem 4) are symmetric monoidal categories.

\section{REFERENCES}

1. J. F. Adams, Stable homotopy and generalized homology, notes by R. Ming, Univ. of Chicago Math. Lecture Notes, 1971 (mimeographed).

2. J. M. Boardman, Stable homotopy theory, University of Warwick, 1965 onwards (mimeographed).

3. J. M. Boardman and R. M. Vogt, Homotopy-everything H-spaces, Bull. Amer. Math. Soc. 74 (1968), 1117-1122. MR 38 \#5215.

4. S. Eilenberg and G. M. Kelly, Closed categories, Proc. Conf. Categorical Algebra (La Jolla, Calif., 1965), Springer, New York, 1966, pp. 421-562. MR 37 \# 1432.

5. H. M. Hastings, A smash product for spectra, Bull. Amer. Math. Soc. 79 (1973), 946-952.

6. A. Heller, Stable homotopy categories, Dull. Amer. Math. Soc. 74 (1968), 28-63. MR $36 \# 7137$.

7. J. P. May, The stable homotopy category (to appear).

8. D. Puppe, On the stable homotopy category, Math. Institut, Heidelberg, 1972 (mimeographed).

9. E. H. Spanier and J. H. C. Whitehead, Duality in homotopy theory, Mathematik a 2 (1955), 56-80. MR 17, 653.

10. R. Vogt, Boardman's stable homotopy category, Aarhus Universitet Lecture Notes, no. 21, 1971 (mimeographed). 11550

D EPARTMENT OF MATHEMATICS, HOFSTRA UNIVERSITY, HEMPSTEAD, NEW YORK

Current address: Department of Mathematical Sciences, SUNY at Binghamton, Binghamton, New York 13901 